\title{
Data reduction of friction factor, permeability and inertial coefficient for a compressible gas flow through a milli-regenerator.
}

\author{
Emna DELLALI ${ }^{1}$, François LANZETTA ${ }^{1, *}$, Sylvie BEGOT ${ }^{1}$, Eric GAVIGNET ${ }^{1}$, and \\ Jean-Yves RAUCH ${ }^{2}$ \\ ${ }^{1}$ FEMTO-ST Institute, Univ. Bourgogne Franche-Comté, CNRS, Energy Department, 90000 Belfort, \\ France \\ 2 FEMTO-ST Institute, Univ. Bourgogne Franche-Comté, CNRS, AS2M Department, 25000 \\ Besançon, France
}

\begin{abstract}
A regenerator of a Stirling machine alternately absorbs and releases heat from and to the working fluid which allows to recycle rejected heat during theoretical isochoric processes. This work focuses on a milli-regenerator fabricated with a multiple jet molding process. The regenerator is a porous medium filled with a dense pillar matrix. The pillars have a geometrical lens shape. Two metallic layers (chromium and copper) are deposited on the polymer pillars to increase heat transfer inside the regenerator. We performed experiments on different milli-regenerators corresponding to three porosities $(\varepsilon=0.80,0.85$ and 0.90$)$ under nitrogen steady and oscillating compressible flows (oscillating Reynolds number in the range $0<R e_{\omega}<60$ and Reynolds number based on the hydraulic diameter $\left.R e_{D h, \max }<6000\right)$ for different temperature gradients $\left(\Delta T<100^{\circ} \mathrm{C}\right)$. Temperature, velocity and pressure experimental measurements are performed with microthermocouples (type $\mathrm{K}$ with 7,6 $\mu \mathrm{m}$ diameter), hotwires and miniature pressure sensors, respectively. We identified a threeterm composite correlation equation for the friction factor based on a Darcy-Forchheimer flow model that best-fit the experimental data. In steady and oscillating flows permeabilities and inertial coefficients are of the same magnitude order. Inertial coefficients decrease when the porosities increase.
\end{abstract}

\section{Introduction}

The regenerator plays a key role on the performances of Stirling machines (engines, coolers, heat pumps). It stores and releases heat during the isochoric displacements. In order to provide high efficiency of the machine, this regenerative heat must be exchanged in a regenerator with a high thermal efficiency. In an ideal Stirling cycle, this efficiency is

* Corresponding author: francois.lanzetta@univ-fcomte.fr 
$100 \%$ and the thermal efficiency of the machine corresponds to the Carnot efficiency. In this case, all thermodynamic processes are thermodynamically reversible, there is no pressure drop in the regenerator, not heat conduction loss from the warm end to the cold end, there is an infinite rate of heat transfer between the working gas and solid matrix and the solid matrix presents an infinite heat capacity. In reality, the regenerator efficiency is less than $100 \%$ and the fluid flow across the regenerator creates a pressure drop and the heat transfer between the gas and the solid matrix are not reversible and infinite. A regenerator is a solid porous matrix with a high surface ratio in order to increase the heat transfer with the working gas. To increase the heat transfer, the speed of the fluid is also increased. But a high velocity flow increases the pressure losses and finally decreases the performance of the regenerator. The regenerator design must be optimized for each gas (Nitrogen, Helium, Hydrogen) considering different geometrical parameters (length, porosity, hydraulic diameter) and thermal parameters (temperature gradient between the two ends, thermophysical properties of the solid matrix).

Many experimental and numerical work has been performed in order to analyse the performances of regenerators [1-5]. In Computational Fluid Dynamics analysis, the regenerator can be modelled using commercial codes (ANSYS FLUENT, Star CCD, Open Foam) upon different numerical methods (SIMPLE, PISO) $[1,6]$. The regenerator is divided into several sections (from three to five) and the results are focused on spaces and time variations of the temperature gradient along the regenerator and heat transfer between the gas and the solid matrix. Experimental works concern flow resistances and heat transfer characteristics of regenerators with different geometrical forms and materials (wire mesh, metal foams, microchannels, involute foils, sponge metals). Authors performed experiments to determine the friction factors (the Darcy coefficients, the Forchheimer inertial coefficients). Tanaka et al. [7] obtained the efficiency and heat transfer coefficient by measuring the pressure drops and the periodic temperature variation at both ends of the regenerator (wires, sponge metal). They determined the friction factor empirical relationship based on hydraulic diameter considered as a representative length. Ishii et al. [8] determined flow characteristics and pressure drop for matrix composed of sintered metal fiber with high porosity. They obtained an empirical equation for the friction factor function of the hydraulic diameter and the porosity of the matrix. They formulated an empirical equation for the Nusselt number function of the Reynolds number and they estimated the reheat loss and thermal efficiency. Kumar et al. [9] proposed a correlationbased methodology to quantify the Darcy permeability and Forchheimer inertial coefficient of porous structures for Stirling cryocoolers. They performed a CFD simulation model (2D axis-symmetric) with Ansys Fluent and determined the pressure drops at different massflow rates at both ends of a regenerator. They identified and compared a correlation to reported experimental pressure drop. Landrum et al. [10] calculated Darcy permeability and inertial coefficients for different mesh screens from CFD simulations compared to experimental study.

In this study, we will determine the permeability and inertial coefficients for unidirectional and oscillating compressible gas flows. We will identify semi-empirical correlations from experimental measurements performed in milli-regenerators of different porosity. 


\section{Materials and methods}

\subsection{Experimental setup}

\subsubsection{Fabrication of the milli-regenerator}

An additive manufacturing process, a Multiple Jet Molding, associated to a 3D printer ProJet ${ }^{\mathrm{TM}} \mathrm{HD} 3500$ is used to produce the different geometries of regenerators. The laminating pitch is $0.2 \mathrm{~mm}$. The thickness of the layers deposited is $32 \mu \mathrm{m}$ for a resolution of $375 \times 375 \times 790$ DPI (X Y Z). The precision of the machine is of the order of $0.1-0.2 \%$ of the dimensions of the printed object. The material is a UV-curable acrylate polymer of the type "Visijet 39 Crystal and their thermophysical properties are presented in the Table 1. Each regenerator consists of a cylinder with a rectangular channel with a matrix of pillars runs through. Two of three regenerators with different porosities $(\varepsilon=0.85$ and 0.90$)$ are presented in Figure 1.

Table 1. Thermophysical properties of the regenerator material.

\begin{tabular}{|c|c|c|c|c|}
\hline $\begin{array}{c}\text { Density } \\
\boldsymbol{\rho}\left(\mathbf{k g} \cdot \mathbf{m}^{-\mathbf{3}}\right)\end{array}$ & $\begin{array}{c}\text { Thermal conductivity } \\
\boldsymbol{\lambda}\left(\mathbf{W} \cdot \mathbf{m}^{-\mathbf{1}} \cdot \mathbf{K}^{-\mathbf{1}}\right)\end{array}$ & $\begin{array}{c}\text { Specific heat } \\
\mathbf{c}_{\mathbf{p}}\left(\mathbf{J} \cdot \mathbf{k g}^{-\mathbf{1}} \cdot \mathbf{K}^{-\mathbf{1}}\right)\end{array}$ & $\begin{array}{c}\text { Diffusivity } \\
\mathbf{a}\left(\mathbf{m}^{\mathbf{2}} \cdot \mathbf{s}^{\mathbf{- 1}}\right)\end{array}$ & $\begin{array}{c}\text { Effusivity } \\
\mathbf{b}\left(\mathbf{J} \cdot \mathbf{K}^{-1} \mathbf{m}^{-\mathbf{2}} \cdot \mathbf{s}^{\mathbf{1} / \mathbf{2}}\right)\end{array}$ \\
\hline 1064 & 0.216 & 1670 & $1.22 \times 10^{-7}$ & 620 \\
\hline
\end{tabular}
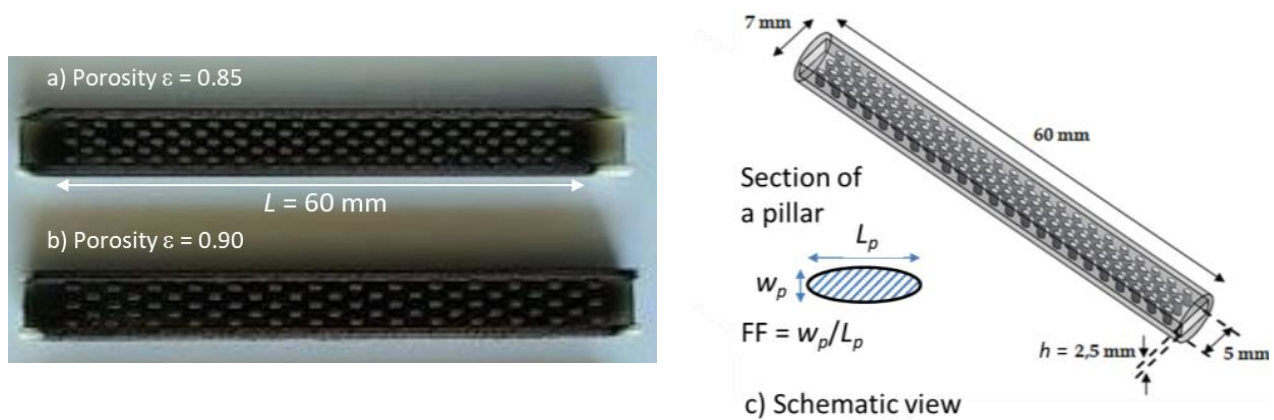

Fig. 1. Milli-regenerator structures made by Multiple Jet Molding (Length $L=60 \mathrm{~mm}$, free internal section $2.5 \times 5 \mathrm{~mm}^{2}$, a) Porosity $\varepsilon=0.85$, b) Porosity $\varepsilon=0.90$ and c) Schematic view)

The regenerator is a porous medium with a total length $L=60 \mathrm{~mm}$ filled with a dense pillar matrix. The pillars have a geometrical lens shape with a form factor $F F=0.5$ (aspect ratio width $w_{p}$ /length $L_{p}$ ) and a height $h=2.5 \mathrm{~mm}$. The free internal section is $5 \times 2.5 \mathrm{~mm}^{2}$. Two metallic layers (chromium and copper) with a total thickness of $800 \mathrm{~nm}$ are deposited on the polymer pillars to increase heat transfer inside the regenerator.

\subsubsection{Test bench}

The experimental setup is shown in Fig. 2. An electric motor (1) drives two pistons (2 and 3 ) by means of a connecting rod/crank mechanism to generate the alternating flow (Fig. 2a). In our experiments, the angle between the two pistons is set to $180^{\circ}$ in order to generate only an alternating transfer flow between the two volumes of the chambers. The rotational speed of the electric motor varies between 10 to $600 \mathrm{rpm}$. The stroke of each piston varies between 5 and $25 \mathrm{~mm}$ and the inner diameter of the cylinder of each chamber 
is $40 \mathrm{~mm}$. The reference crank angle is set to $0^{\circ}$ when the cold side piston is at its Bottom Dead Centre (BDC) and the hot side piston at its Top Dead Centre (TDC) (Fig. 2b). The milli-regenerator (6) is in the middle of the setup between two heat exchangers (4 and 5). These heat exchangers can be cooled (4) or heated (5) by a cold or hot water flow respectively in order to impose a gas temperature gradient $\Delta T$ between the two ends of the milli-regenerator and $0<\Delta T<70^{\circ} \mathrm{C}$. The pressure $\left(P_{1}, P_{2}\right)$, velocity $\left(V_{1}, V_{2}\right)$ and temperature $\left(T_{1}, T_{2}\right)$ of the airflow are measured at both ends of the milli-regenerator. The diameter of the connecting pipes between the heat exchangers and the regenerator is $5 \mathrm{~mm}$.

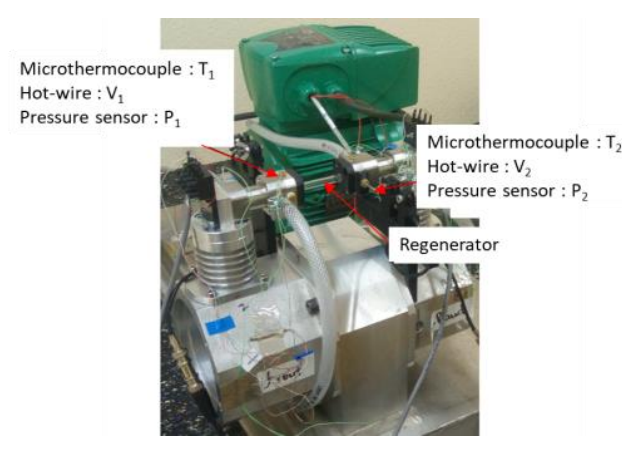

a)

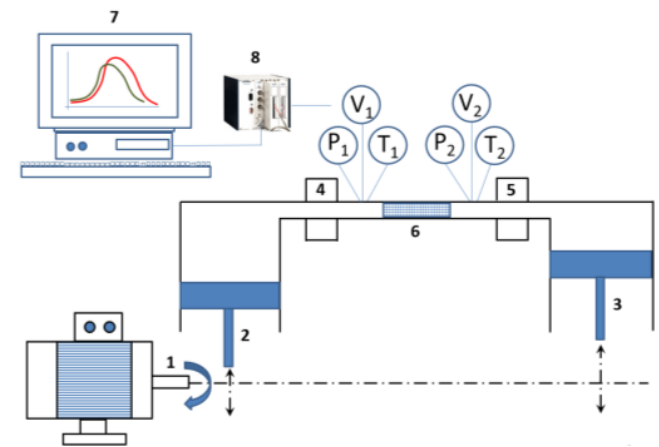

b)

Fig. 2. Experimental setup - 1: Electric motor; 2 and 3: Pistons; 4: Cold Heat Exchanger (CHX); 5: Hot Heat Exchanger (HHX); 6: Milli-regenerator; 7 and 8: Data acquisition system

Gas pressures are measured with ultraminiature pressure transducers (Kulite XCQ-055, 1.7 BARA-8068 and bandwidth is $210 \mathrm{kHz}$ ). They were calibrated using a Drück PV621 Pressure Station within the pressure range $\left[P_{a t m}-3 \mathrm{bar}\right]$. The velocity measurements are achieved with hot wire anemometers (TSI IFA300 range $0.15-200 \mathrm{~m} \cdot \mathrm{s}^{-1}, 600 \mathrm{kHz}$ bandwidth). Each probe measures the axial velocity component and was calibrated in the laboratory. The fluid temperatures are measured with home-made $12.7 \mu \mathrm{m}$ diameter type $\mathrm{K}$ microthermocouples (accuracy $\pm 0.1^{\circ} \mathrm{C}$ and cut-off frequency $30 \mathrm{~Hz}$ ). The static response is not presented in this work but the probes were placed in a regulated oven within the range $\left[30-300^{\circ} \mathrm{C}\right]$ and the measured temperatures were compared with a precision thermometer (Pt100 platinum reference resistance, accuracy $\pm 0.005{ }^{\circ} \mathrm{C}$ ). Each thermocouple presents a Seebeck coefficient of $40 \mu \mathrm{V} /{ }^{\circ} \mathrm{C}$ at $20^{\circ} \mathrm{C}$. The experimental dynamic characterization of the sensors consists in the determination of their temporal and frequency characteristics (response time, cut-off frequency and phase shift of signals) for different types of convective excitations in non-stationary regimes [11]. The measured signals are registered with the data acquisition system (7 and 8) operating at $200 \mathrm{kHz}$ maximum sample rate.

\subsection{Uncertainty analysis}

We followed the method presented in the Guide to the expression of Uncertainty in Measurement (GUM) to determine the experimental uncertainties [12]. The pressure and velocity measurement are the average of 40 successive cycles. The maximal type A uncertainties are observed at the lowest frequency $(2 \mathrm{~Hz})$, they were of $0.022 \mathrm{~m} . \mathrm{s}^{-1}$ or $1.45 \%$ for the maximal velocity at this frequency and $8.75 \times 10^{-5}$ bar or $1.48 \%$ for the maximal pressure drop at this frequency. This worst-case uncertainty is used in the calculation of the total uncertainty. For the type B uncertainties, we use the supplier 
characteristics of the calibration apparatus for the velocity and pressure (resp. $+/-2 \%$ and $+/-2.5 \times 10^{-4}$ bar). The total relative velocity uncertainty varies from $3.7 \%$ at $2 \mathrm{~Hz}$ to $2.4 \%$ at $10 \mathrm{~Hz}$. The relative pressure uncertainty varies from $5.7 \%$ at $2 \mathrm{~Hz}$ to $3.9 \%$ at $10 \mathrm{~Hz}$. The temperature sensors are calibrated with a reference probe and the uncertainty is $+/-0.1^{\circ} \mathrm{C}$.

We consider that the uncertainty in the thermophysical properties due to the temperature measurement uncertainty is small compared to the other uncertainties. The dimensions uncertainties are $0.2 \%$ due to the resolution of the $3 \mathrm{D}$ printer. The porosity uncertainty is assumed to be $1 \%$. The errors are supposed uncorrelated. Therefore, applying the law of propagation of uncertainties leads to relative uncertainties for the Reynolds number, pressure drop and friction factor. The relative uncertainties depend on the frequency. They vary from $3.7 \%$ to $2.6 \%$ for the Reynolds number, from 5.7 to $0.4 \%$ for the pressure drop, and for the friction factor, from $7.8 \%$ to $3.6 \%$.

\section{Results and discussions}

We performed experiments on different milli-regenerators with three porosities $(\varepsilon=0.80$, 0.85 and 0.90 ) under nitrogen steady and oscillating flows (oscillating Reynolds number in the range $0<R e_{\omega}<60$ and Reynolds number based on the hydraulic diameter $R e_{D h}<6000$ ) for different temperature gradients $\left(\Delta T<100^{\circ} \mathrm{C}\right)$.

\subsection{Steady flow}

A continuous controlled mass flow of nitrogen passes through the regenerator. The maximal pressure drop $\Delta P$ is measured as a function of the Reynold number $R e_{D h}$ based on the hydraulic diameter $D_{h}$ with $R e_{D h}<6000$ (Fig. 3). Steady flow can be considered as the zero-frequency limit of oscillating flow that produces the same mass flow.

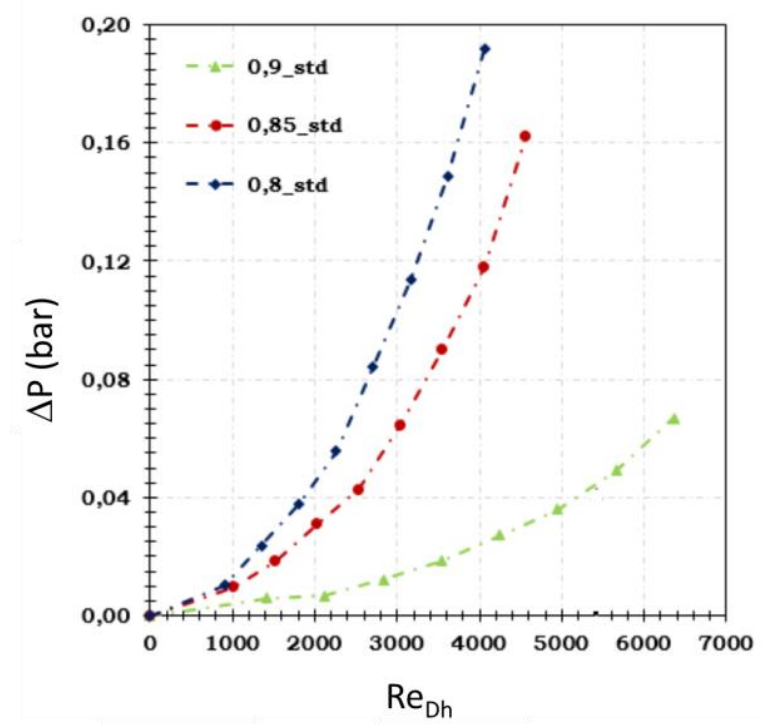

Fig. 3. Maximal pressure drop $\Delta P$ for a steady unidirectional flow versus Reynolds number $R e_{D h}$ 
Darcy's law requires correction when the particle-diameter-based Reynolds number exceeds unity. Darcy's law means that the drag is linearly proportional to the velocity. In the fluid momentum equation written for compressible flow, the permeability $K$ and the inertial coefficient $F$ need to be evaluated for each kind of regenerator porous medium. Vafai and Tien [13] and Pamuk and Ozdemir [14] presented semi-empiric correlation equations for fluid flow through packed bed of spherical balls based on the model of Ergun [15]. Even if the milli-regenerator is filled with pillars (not spherical balls), we consider a quadratic relation for pressure drop such as the Darcy-Forchheimer quasi steady-flow form of the 1-D fluid momentum equation $[16,17]$ :

$$
\frac{\Delta P}{L}=\frac{\mu V}{K}+\frac{F}{\sqrt{K}} \rho V^{2}
$$

This equation represents the balance between the two drag forces and the pressure force needed to overcome them, to maintain average velocity in the porous medium. Considering maximal pressure drop $\Delta P$, Reynolds number $\operatorname{Re}_{\mathrm{Dh}}$, permeability $K$ and inertial coefficient $F$, equation (1) becomes:

$$
\frac{\Delta P}{L} \frac{D_{h}^{2}}{\mu V}=\frac{D_{h}^{2}}{K}+\frac{F D_{h}}{\sqrt{K}} R e_{D h}
$$

This equation is written in terms of the Darcy friction factor $C_{f}$ for steady flows in microchannels $[14 ; 16-21]$ as follows :

$$
C_{f}=\frac{\Delta P}{\frac{\rho V^{2}}{2} \frac{L}{D_{h}}}=\frac{2 \frac{D_{h}^{2}}{K}}{R e_{D h}}+\frac{2 F D_{h}}{\sqrt{K}}
$$

Friction factor $C_{f}$ correlations for various type of regenerator materials (random fiber, wire screen, microchannels, involute foils) are of the general form we based on hydraulic diameter $D_{h}[7,16]$ :

$$
C_{f, s t d}=\frac{a_{s t d}}{R e_{D h}}+b_{s t d} R e_{D h}^{c}
$$

Equation (4) is a three-term composite correlation equation that best-fit the experimental data in steady flow. We define the friction factor $C_{f, s t d}$, the permeability $K_{s t d}$ and the inertial coefficient $F_{\text {std }}$ :

$$
C_{f, s t d}=\frac{2 \frac{D_{h}^{2}}{K_{s t d}}}{R e_{D h}}+\frac{2 F_{s t d} D_{h}}{\sqrt{K_{s t d}}}
$$

The coefficients $K_{\text {std }}$ and $F_{\text {std }}$ are obtained from Equations (2) to (5) and:

$$
K_{s t d}=2 \frac{D_{h}^{2}}{a} \quad \text { and } \quad F_{s t d}=\frac{b}{\sqrt{2 a}} R e_{D h}^{c}
$$

The three constants $a, b$ and $c$ are identified from experimental data [22]. Figures 4a,b,c,d show the friction factor after constants identification (Tab. 4). For low Reynolds 
number $(\operatorname{Re}<2000)$, results are not logical because the friction factors are lower in high porosities in low Reynolds numbers. The pressure uncertainties are higher at low Reynolds number and consequently could impact the numerical values of friction factors due to the propagation of uncertainties.

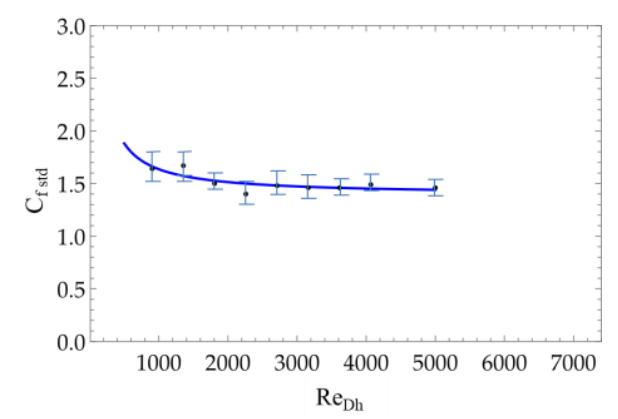

a) Porosity $\varepsilon=0.80 \_$std

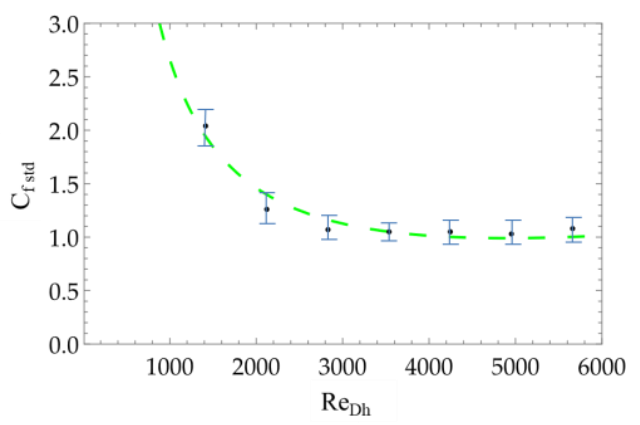

c) Porosity $\varepsilon=0.90 \_$std

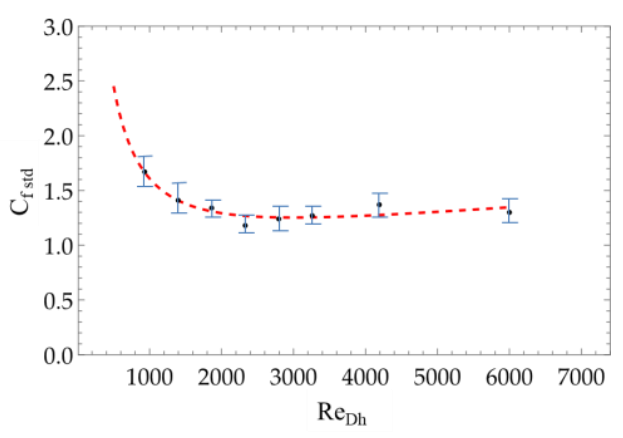

b) Porosity $\varepsilon=0.85 \_$std

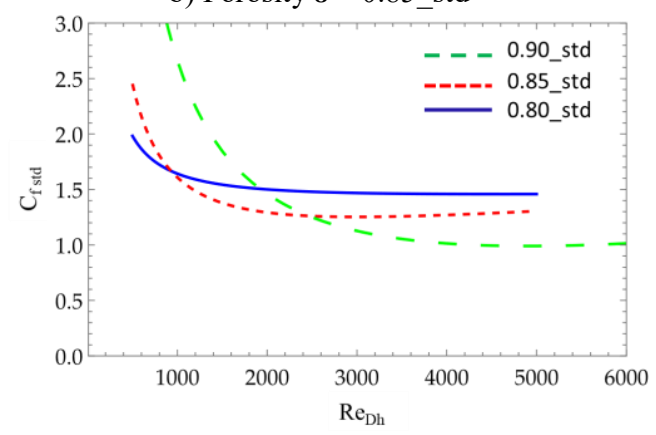

d) Superposed models

Fig. 4. Friction factor $C_{f, s t d}$ for the three regenerator porosities $\left(\varepsilon=0.90 \_\right.$std $; 0.85 \_$std and $0.80 \_$std respectively) in steady unidirectional flow versus Reynolds number

Results indicate that the inertial coefficient $F_{\text {std }}$ of the compressible gas in steady flow decreases when the porosity increases and, for each porosity, increases with the Reynolds number $\operatorname{Re}_{D h}$, (Tab. 2).

Table 2. Friction factor coefficients, permeability $K$ and inertial coefficient $F$ for the regenerators at steady flow.

\begin{tabular}{|c|c|c|c|c|c|c|c|}
\hline \multirow{2}{*}{ Porosity } & \multirow{2}{*}{$\begin{array}{c}\text { Hydraulic } \\
\text { diameter } \\
D_{h}(\mu \mathrm{m})\end{array}$} & \multirow{2}{*}{$a$} & \multirow{2}{*}{$b$} & \multirow{2}{*}{$c$} & \multirow{2}{*}{$\begin{array}{l}\text { Permeability } \\
K_{\text {std }}\left(\mathbf{m}^{2}\right)\end{array}$} & \multicolumn{2}{|c|}{ Inertial coefficient $\boldsymbol{F}_{\text {std }}$} \\
\hline & & & & & & $R e_{D h}=2500$ & $R e_{D h}=5000$ \\
\hline$\varepsilon=0.80$ & 268 & 392.2 & 0.8181 & 0.0614 & $3.6626 \times 10^{-10}$ & $47.22 \times 10^{-3}$ & $49.28 \times 10^{-3}$ \\
\hline$\varepsilon=0.85$ & 445 & 980.7 & 0.0547 & 0.3532 & $4.0384 \times 10^{-10}$ & $19.58 \times 10^{-3}$ & $25.01 \times 10^{-3}$ \\
\hline$\varepsilon=0.90$ & 622 & 2589.3 & 0.000027 & 1.1448 & $2.9980 \times 10^{-10}$ & $2.966 \times 10^{-3}$ & $6.558 \times 10^{-3}$ \\
\hline
\end{tabular}


In order to compare results with steady flows, we conducted experiments in oscillating flows with oscillating Reynolds number $R e_{\omega}=\omega D_{h}{ }^{2} / v$ the range $0<R e_{\omega}<60$ for different temperature gradients $\left(\Delta T<100^{\circ} \mathrm{C}\right)$, based on the hydraulic diameter and for $\operatorname{Re}_{D h}<6000$. The exchangers are temperature-controlled and keep a temperature gradient between the two ends of the regenerator of $\Delta T=30^{\circ} \mathrm{C}, 40^{\circ} \mathrm{C}$ and $50^{\circ} \mathrm{C}$. The strokes of the pistons are $S$ $=24 \mathrm{~mm}$ and $S=30 \mathrm{~mm}$. The different tests were made for five frequencies: 2, 4, 6, 8 and $10 \mathrm{~Hz}$. Figure 5 shows the principle of measurement acquisition (Fig. 2). From 0 to $180^{\circ}$, the fluid leaves the cold exchanger ( $\mathrm{CHX})$ and enters the regenerator: this is the "Cold Blow" phase. Conversely, from $360^{\circ}$ to $180^{\circ}$, the fluid leaves the hot exchanger (HEX) and enters the regenerator: this is the "Hot Blow" phase. Normally we should have negative velocity oscillations from 0 to $180^{\circ}$ and positive from 360 to $180^{\circ}$. On figure 5, these oscillations are positive because the velocity sensor measures only positive quantities. There is a phase shift $\phi$ between the velocity of the piston and that of the fluid. This phenomenon is caused by the inertia of the compressible fluid at each change of direction. We have observed this phase shift $\phi$ varies between $18^{\circ}$ and $22^{\circ}$ depending on the fluid velocity. The phase shift increases as the fluid velocity increases [22].

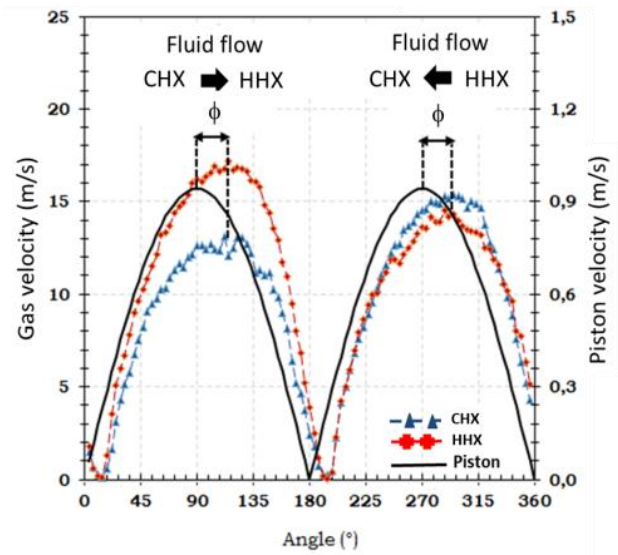

Fig. 5. Friction gas velocity at the hot outlet of the regenerator $(\varepsilon=0.80, S=30 \mathrm{~mm}$, isothermal flow at $22^{\circ} \mathrm{C}, f=10 \mathrm{~Hz}$ )

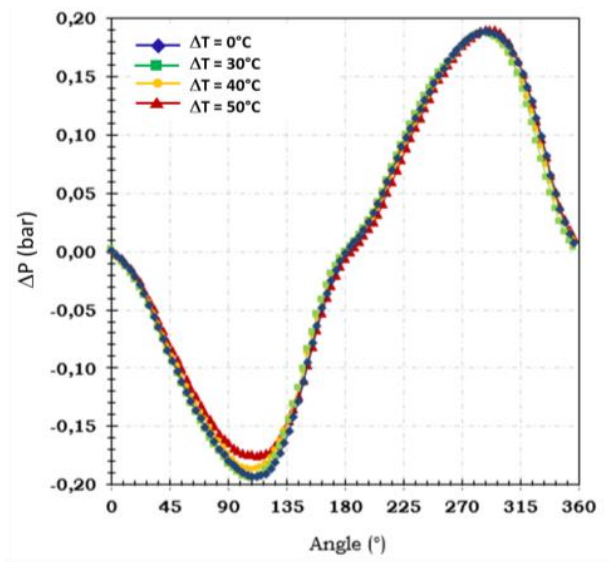

Fig. 6. Influence of the temperature gradient on the pressure drop $\left(\varepsilon=0.80, S=30 \mathrm{~mm}, \Delta \mathrm{T}=0^{\circ} \mathrm{C}\right.$ (isothermal flow), $30^{\circ} \mathrm{C}, \quad 40^{\circ} \mathrm{C}$ and $50^{\circ} \mathrm{C}$ respectively, $f=10 \mathrm{~Hz}$ ).

Figures 5 and 6 show that the maximum velocity occurs when the pressure drop is maximum and the piston is zero. Figure 6 shows the influence of temperature gradients on the pressure drop. It can be seen that the temperature gradient only affects the Cold Blow phase when the fluid reaches its highest negative pressure change (at angle $120^{\circ}$ ). This slight expansion changes the thermophysical properties of the fluid (density and viscosity) enough to create a pressure change. During the Hot Blow phase, the fluid is sufficiently hot and the slight compression (at angle $290^{\circ}$ ) does not generate a sufficiently large overpressure variation. Usually, the temperature gradient between $30^{\circ} \mathrm{C}$ and $70^{\circ} \mathrm{C}$ does not affect the pressure drop. We can now continue the study from the measurements made in isothermal regime for $\Delta T=0^{\circ} \mathrm{C}$.

The rotation frequency changes the pressure drop within the regenerator (Fig. 7). The pressure drop increases with the square of the fluid speed and therefore with the frequency. The inertial effects coupled with the compressibility of the fluid create a phase shift of 
about $22^{\circ}$ between the maximum pressure drops at 2 and $10 \mathrm{~Hz}$ (for the Hob Blow and the Cold Blow periods).

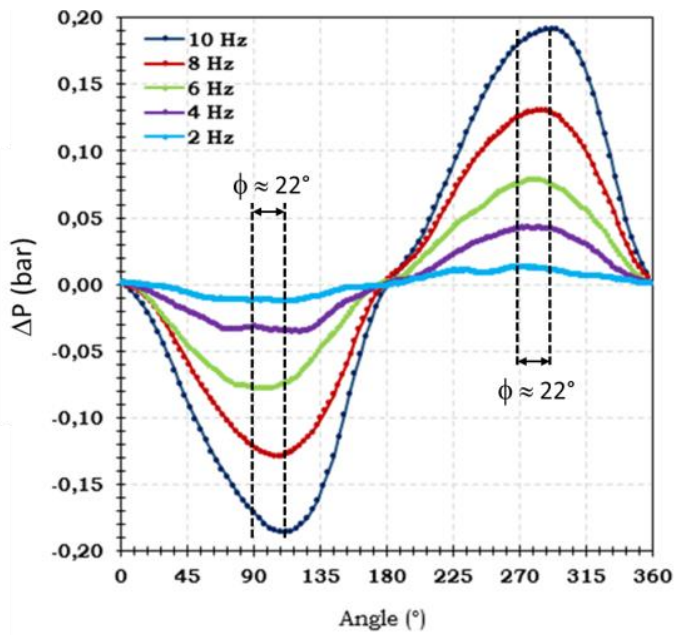

Fig. 7. Pressure drop of the regenerator fluid flow at different frequencies $\left(\varepsilon=0.80 \_\right.$osc, $S=30 \mathrm{~mm}$, isothermal flow $\Delta T=0^{\circ} \mathrm{C}$ )

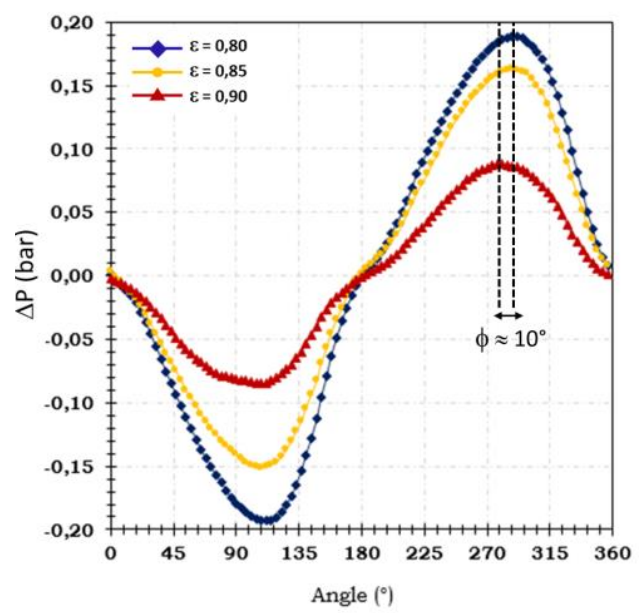

Fig. 8. Influence of the porosity on the pressure drop $\left(\varepsilon=0.80 \_\right.$osc, $f=10 \mathrm{~Hz}, S=30 \mathrm{~mm}$, isothermal flow $\Delta T=0^{\circ} \mathrm{C}$ )

The viscous frictions decrease with the porosity of the regenerator (Fig. 8). Indeed, the greater the porosity, the smaller the contact surface area with the fluid. A slight phase shift of around $10^{\circ}$ is observed between the extreme values of pressure gradient. As the porosity decreases, the effects of compressibility and friction increase and tend to delay the appearance of the pressure peak.

In oscillating flows, velocity, pressure and temperature vary with time and it is obvious that friction factor varies with time too. The fluid velocities are measured at the two extremities of the regenerator at the same time. When the compressible fluid enters the regenerator, there is a compression and, consequently, there is a local decrease of the inlet fluid velocity. At the same time, due to the mass conservation, the fluid leaving the regenerator is expanded and accelerated increasing its turbulence. In order to compare our results with literature, we consider velocity and Reynolds number $R e_{D h \text {, max }}$ corresponding to maximum pressure gradient value $\Delta P_{\max }$ (Fig. 9). Pressures drops increase with Reynolds number and at given Reynolds number decrease as the porosity increases.

According to Eq. (4), the friction factor $C_{f, \text { osc }}$ will be based on the maximal value of the velocity measured on a period of oscillation, i.e. the maximal value of the Reynolds number $R e_{D h, \max }$ :

$$
C_{f o s c}=\frac{a_{o s c}}{R e_{D h, \max }}+b_{o s c} R e_{D h, \max }^{c_{o s c}}
$$

Based on equations (4) and (6), the equivalent oscillating permeability $K_{\text {osc }}$ and inertial coefficient $F_{o s c}$ at maximal Reynolds number $R e_{D h, \max }$ become:

$$
K_{o s c}=2 \frac{D_{h}^{2}}{a_{o s c}} \quad \text { and } \quad F_{o s c}=\frac{b}{\sqrt{2 a_{o s c}}} R e_{D h, \max }^{c}
$$




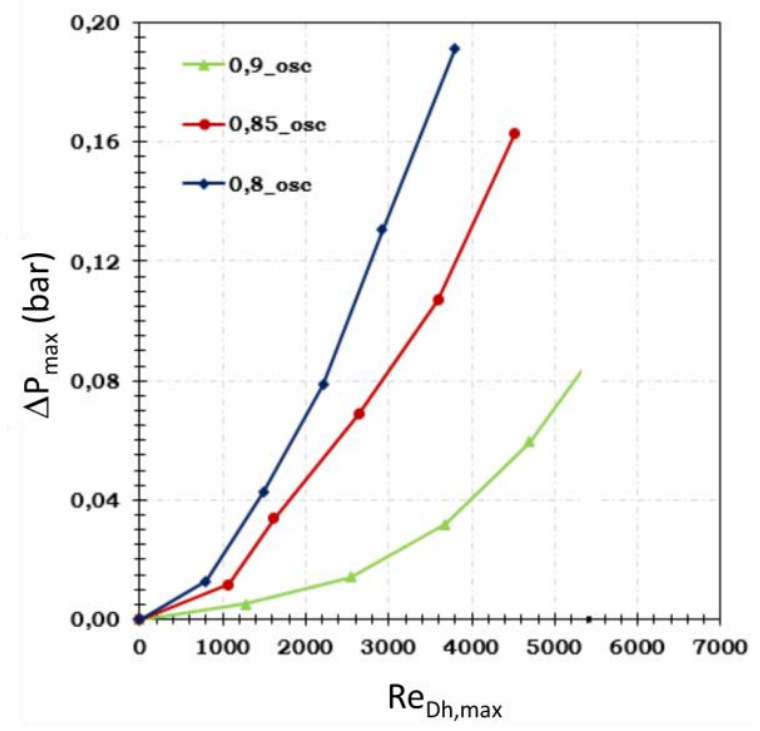

Fig. 9. Maximal pressure drops $\Delta P_{\max }$ for an oscillating flow versus maximal Reynolds number $R_{D h, \max }$

For values of Reynolds numbers between 2500 to 5000 (Fig. 10), friction factor correlations $C_{\text {f,osc }}$ present close values for the two porosities 0.80 osc and 0.85 _osc but differ for the higher porosity $0.90 \_$osc. We cannot conclude for the 0.90 osc porosity because we found there was measurement errors during the experiments. The measurements at $\operatorname{Re}>4500$ were too noisy to be accepted.

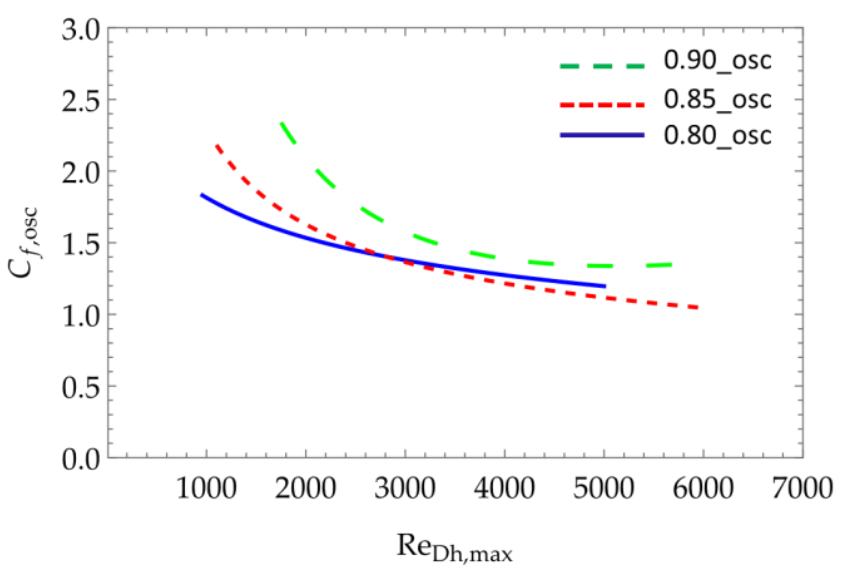

Fig. 10. Friction factor correlations $C_{f \text {,osc }}$ for the three regenerator porosities $\left(\varepsilon=0.90 \_\right.$osc $; 0.85$ osc and 0.80_osc respectively) in oscillating flow versus maximal Reynolds number $R e_{D h \text {, } \max }$ 
Table 3. Friction factor coefficients, permeability $K_{\mathrm{osc}}$ and inertial coefficient $F_{\mathrm{osc}}$ for the regenerators at oscillating flow.

\begin{tabular}{|c|c|c|c|c|c|c|c|}
\hline \multirow[b]{2}{*}{ Porosity } & \multirow{2}{*}{$\begin{array}{c}\text { Hydraulic } \\
\text { diameter } \\
D_{h}(\mu \mathrm{m})\end{array}$} & \multirow{2}{*}{$a_{\text {osc }}$} & \multirow{2}{*}{$b_{\text {osc }}$} & \multirow{2}{*}{$c_{\text {osc }}$} & \multirow{2}{*}{$\begin{array}{l}\text { Permeability } \\
\quad K_{o s c}\left(\mathbf{m}^{2}\right)\end{array}$} & \multicolumn{2}{|c|}{ Inertial coefficient $\boldsymbol{F}_{\text {osc }}$} \\
\hline & & & & & & $\begin{array}{c}R e_{D h, \max } \\
=\mathbf{2 5 0 0}\end{array}$ & $\begin{array}{c}R e_{D h, \max } \\
=\mathbf{5 0 0 0}\end{array}$ \\
\hline$\varepsilon=0.80$ & 268 & 574.5 & 8.428 & -0.2503 & $2.5004 \times 10^{-10}$ & $35.08 \times 10^{-3}$ & $29.49 \times 10^{-3}$ \\
\hline$\varepsilon=0.85$ & 445 & 721.6 & 13.707 & -0.314 & $5.4885 \times 10^{-10}$ & $30.92 \times 10^{-3}$ & $24.89 \times 10^{-3}$ \\
\hline$\varepsilon=0.90$ & 622 & 3832 & 0.000011 & 1.275 & $2.0192 \times 10^{-10}$ & $2.70 \times 10^{-3}$ & $6.54 \times 10^{-3}$ \\
\hline
\end{tabular}

Permeabilities in steady and oscillating flows, $K_{s t d}$ and $K_{\text {osc }}$ respectively, are of the same magnitude order (from $2.10^{-10}$ to $5.10^{-10} \mathrm{~m}^{2}$ ) and $0.66<K_{\text {osc }} / K_{\text {std }}<1.37$. In steady and oscillating flows, the inertial coefficients $F_{\text {osc }}$ are of the same order and decrease when the porosity increases for Reynolds number greater than 2500. For the lower values of Reynolds number, we cannot conclude because of the uncertainties concerning the pressure measurements.

\section{Conclusion and perspectives}

Gaseous flow characteristics of nitrogen in milli-regenerators were studied. Each regenerator, fabricated with a Multiple Jet Molding process, is a cylinder with a rectangular channel with a matrix of pillars with three porosities $(\varepsilon=0.80,0.85$ and 0.90$)$. The regenerator is a porous medium with a total length $L=60 \mathrm{~mm}$ and filled with a dense pillar matrix. The pillars have a geometrical lens shape with a form factor $F F=0.5$ (aspect ratio width/length) and a height $h=2.5 \mathrm{~mm}$. Two metallic layers (chromium and copper) with a total thickness of $800 \mathrm{~nm}$ are deposited on the polymer pillars to increase heat transfer inside the regenerator. The regenerators were tested under nitrogen steady flows and oscillating flows (oscillating Reynolds number in the range $0<R e_{\omega}<60$ and Reynolds number based on the hydraulic diameter $R e_{D h}<6000$ ) for different temperature gradients $\left(\Delta T<100^{\circ} \mathrm{C}\right)$. The major findings can be drawn as follows.

In steady flow we identified a three-term composite correlation equation for the friction factor based on a Darcy-Forchheimer flow model that best-fit the experimental data. The inertial coefficient $F_{\text {std }}$ of the compressible gas decreases when the porosity increases and increases with the Reynolds number $R e_{D h}$ for each porosity. The steady flow show permeability coefficient of the same magnitude order.

In oscillating flow, the compressibility and inertia of the gas affect the characteristics of the oscillating flow. A phase shift occurs between the velocity of the piston and the velocity of the gas. The maximum gas velocity is delayed by an angle varying from $18^{\circ}$ to $22^{\circ}$ with respect to the maximum piston velocity. Similarly, as the frequency of rotation increases, the pressure maxima are shifted by an angle of about $22^{\circ}$. The temperature of the gas (between $30^{\circ} \mathrm{C}$ and $70^{\circ} \mathrm{C}$ ) do not affect the pressure oscillations in the experimental range of the regenerator. We have adopted the same kind of friction coefficient correlation as for the steady flow. The Reynolds number $R e_{D h, \max }$ corresponds to the maximal velocity obtained in an oscillating cycle, at the maximal pressure of the flowing gas. The inertial coefficients $F_{\text {osc }}$ are of the same magnitude order than in steady flows and decrease when the porosities increase.

Improving the performance of the regenerator requires characterizing its behavior under different materials constraints (polymer and deposited metals), geometrical constraints 
(pillar shape, porosity, form factor), thermal constraints (low temperature range, thermal efficiency during heat accumulation/restitution) and fluidic constraints (flow regime, pressure drop, compressibility). In this study, we tested only nitrogen as a working fluid. In the future, we will test other gases (helium, hydrogen), other forms of pillars (rhombus, elongated and rectangular). We have started a numerical study (CFD) of the oscillating flow inside a milli-regenerator and we will compare the numerical and experimental results in order to optimize a Stirling machine at small scale and small temperature gradient.

\section{Acknowledgment}

This work has been supported by the EIPHI Graduate School (contract ANR-17-EURE-0002) and the Region Bourgogne-Franche-Comté

\section{References}

1. R. W. Dyson, S. M. Geng, R. C. Tew et M. Adelino, Eng. Appl. of Comput. Fluid Mech. 2, 95, (2008).

2. M. B. Ibrahim et R. C. Tew Jr, Stirling Convertor Regenerators, CRC Press, (2011).

3. R. Gheith, F. Aloui et S. Ben Nasrallah, Fluids Engineering Division Summer Meeting, (2015).

4. S. M. Sadrameli, Renew. and Sust. En. Reviews, 58, (2016).

5. Y. Cui, J. Qiao, B. Song, X. Wang, Z. Yang, H. Li et W. Dai, Energy, 121287, (2021).

6. P. Bitsikas, E. Rogdakis et G. Dogkas, Therm. Sci. and Eng. Prog., 17, 100492, (2020).

7. M. Tanaka, I. Yamashita et F. Chisaka, JSME Int. J.. Ser. 2, Fluids Eng., Heat transfer, Power, Comb. Therm. Prop., 33, (1990).

8. H. Ishii, D. Bouzawa et K. Hamaguchi, Proc. 17th ISEC, 201, (2016).

9. V. V. Kishor Kumar et B. T. Kuzhiveli, Int. J.of Air-Conditioning and Refrig., 25, 1750014, (2017).

10. E. C. Landrum, T. J. Conrad, S. M. Ghiaasiaan et C. S. Kirkconnell, Cryogenics, 50, 373-380, (2010).

11. F. Lanzetta et E. Gavignet, Thermal Measurements and Inverse Techniques, 95, (2011).

12. I. E. C. Bipm, I. IFcc et I. Iso, IUPAP and OIML, Evaluation of measurement data Supplement, 1, (2008).

13. K. Vafai et C. L. Tien, Int. J. Heat and Mass Transfer, 24, 195, (1981).

14. M. T. Pamuk et M. Özdemir, Experim. Therm. Fluid Sci., 38, 134, (2012).

15. S. Ergun, Chem. Eng. Prog., 48, 89, (1952).

16. D. Gedeon et J. G. Wood, NTRS, NASA, (1996).

17. M. Kaviany, Principles of Heat Transfer in Porous Media, Second Edition, Springer, (2005).

18. P. Cheng et T. Zhao, Annual Rev. Heat Transf., 9, (1998).

19. T. S. Zhao et P. Cheng, Cryogenics, 36, 333, (1996).

20. K. C. Leong et L. W. Jin, Int. J. Heat Mass Transfer, 48, 243, (2005).

21. C.-T. Hsu, H. Fu et P. Cheng, J. Fluid. Eng. 121, 52, (1999).

22. E. Dellali, S. Bégot, F. Lanzetta, E. Gavignet et J.-Y. Rauch, Exp. Thermal, Fluid Sci., 103, 394, (2019). 


\section{Nomenclature}

$\begin{array}{ll}a, b, c & \text { constants } \\ \mathrm{BCD} & \text { Bottom Dead Center } \\ c_{p} & \text { specific heat }\left(\mathrm{J} \mathrm{kg}^{-1} \cdot \mathrm{K}^{-1}\right) \\ C & \text { factor } \\ C H X & \text { Cold Heat eXchanger } \\ D & \text { diameter }(\mathrm{m}) \\ E & \text { effusivity }\left(\mathrm{J} \cdot \mathrm{K}^{-1} \cdot \mathrm{m}^{-2} \cdot \mathrm{s}^{-1 / 2}\right) \\ f & \text { frequency }(\mathrm{Hz}) \\ F & \text { inertial coefficient } \\ F F & \text { form factor } \\ \mathrm{GUM} & \text { Guide to the expression of } \\ & \text { Uncertainty in Measurement } \\ h & \text { height }(\mathrm{m}) \\ \mathrm{HHX} & \text { Hot Heat } \mathrm{eXchanger} \\ K & \text { permeability }\left(\mathrm{m}^{2}\right) \\ L & \text { length }(\mathrm{m}) \\ P & \text { pressure }(\text { bar }) \\ R e & \text { Reynolds number } \\ S & \text { stroke }(\mathrm{m}) \\ T & \text { temperature }\left({ }^{\circ} \mathrm{C}\right) \\ \text { TDC } & \text { Top Dead Center }\end{array}$

$\begin{array}{ll}V & \text { velocity }\left(\mathrm{m} \cdot \mathrm{s}^{-1}\right) \\ w & \text { width }(\mathrm{m})\end{array}$

\section{Subscripts}

atm atmospheric

$h \quad$ hydraulic diameter

$f$ friction

$\max$ maximal value

osc oscillating

$p \quad$ pillar

std steady

\section{Greek Symbols}

$\Delta \quad$ difference

$\varepsilon \quad$ porosity

$\lambda$ thermal conductivity $\left(\mathrm{W} \cdot \mathrm{m}^{-1} \cdot \mathrm{K}^{-1}\right)$

$\mu \quad$ dynamic viscosity (Pa.s)

$v \quad$ cinematic viscosity $\left(\mathrm{m}^{2} \cdot \mathrm{s}^{-1}\right)$

$\rho \quad$ density $\left(\mathrm{kg} \cdot \mathrm{m}^{-3}\right)$

$\omega \quad$ angular frequency $\left(\mathrm{rad} . \mathrm{s}^{-1}\right)$ 